\title{
LA INTERVENCIÓN PSICOSOCIAL ANTE EL MALTRATO EN LA NIÑEZ TEMPARANA: ¿ESCUCHA O CONTROL?
}

\author{
Rosa Figueroa Sánchez ${ }^{1}$
}

\section{Resumen}

La aproximación ante el problema del maltrato infantil se afronta desde la contradicción o sea desde la escucha y la coerción, desde la terapia y el control. Este transita por bordes que oscilan entre los contextos legales, sociales y emocionales. El contexto legal configura reglas que definen nuestro modo de actuar como profesionales de la conducta con el fin de proveerle a la niñez protección inmediata. Dados estos bordes nos preguntamos: ¿Cómo le facilitamos a una familia la externalización de una demanda de escucha si esta no la ha pedido?, ¿Qué significado toma para los miembros de la familia la violencia hacia un niño o niña?, ¿Qué enfoque teórico permite el proceso terapéutico con estas familias? El presente trabajo pretende examinar la intervención psicosocial desde un enfoque psicodinámico y reflexionar sobre el papel de la escucha reconociendo que en el tránsito de la palabra de un padre o madre maltratante algo habrá de producirse. Esta escucha se realiza a partir de la experiencia de jugar prestando atención a la relación vincular.

Palabras claves: maltrato infantil, proceso terapéutico, juego, relación vincular.

\section{Abstract}

Through this article the problem of child abuse is approached from the contradiction present in the relations of listening and coercion, and therapy and control. These relations are present in the legal, social and emotional

\footnotetext{
${ }^{1}$ Instructora en la Escuela Graduada de Trabajo Social Beatriz Lassalle, Universidad de Puerto Rico en Río Piedras y Trabajadora Social en el Centro Pediátrico de Servicios Habilitativos, Centro Médico, Puerto Rico.
} 
contexts. The legal context comprises the rules which define our way of responding as behavior professionals with the purpose of providing children with immediate protection. Thus we ask: How do we facilitate for a family the externalization of a demand to be listened to if they have not asked for it? What meanings do the violence against a child takes for family members? What theoretical approach allows for a therapeutically process for these families? The intention of the article is to examine the psychosocial intervention from a psychodynamic approach, and to reflect on the role of listener, acknowledging that in the expressions of an abusive father or mother something must be constructed. This listening is realized from the start point of the experience of playing paying attention to the attachment relationship.

Keywords: child abuse, therapeutic process, play therapy, attachment relationship.

\section{Introducción}

El objetivo central de este artículo es reflexionar sobre el abordaje psicosocial que he realizado desde la perspectiva de la escucha, en que se asume el impacto que tiene el control en el proceso terapéutico. Partimos de lo que exponen Linares y Coletti (1997) respecto a que la percepción de los padres y madres es que están siendo controlados y juzgados más que ayudados. En la comunidad académica han proliferado las investigaciones y las publicaciones sobre el maltrato infantil (Ruiz y Gallardo, 2002; Di Lauro, 2004; Allin et al., 2005; Suprina y Chang, 2005; Bear y Martínez, 2006; Elliot y Urquiza, 2006). Los estudios e investigaciones han girado sobre el impacto del maltrato en niños y niñas, en determinar los factores personales, familiares, relacionales, sociales o económicos que intervienen en la desprotección infantil y los acercamientos terapéuticos mas apropiados (Barudy, 1998).

En las investigaciones examinadas hubo un énfasis en que el maltrato infantil ocurre como una evidencia de la perturbación de las personas adultas que asumen la función paterna y materna con la niña o el niño. Esto llevó a que las explicaciones iniciales sobre el maltrato se vincularán a desórdenes psicopatológicos (Ruiz y Gallardo, 2002). A su vez, repercutió en mantener perspectivas explicativas unicausales que analizaban las características de personalidad de los adultos maltratantes, la transmisión generacional de la conducta de malos tratos o las pautas de crianza, sin articular el significado 
del contexto sociocultural (Elliot y Urquiza, 2006). La perspectiva explicativa psicopatológica produjo un sujeto culpable y una visión esteriotipada sobre éste. El adulto maltratante es visto por la comunidad como un abusador.

La función protectora del rol parental de modo general recae según las normas de nuestra cultura patriarcal en la mujer. En la expectativa de roles que se tiene de la mujer, se espera que esta proteja al niño y niña de cualquier peligro, daño o sufrimiento. Por otro lado, las intervenciones han enfatizado en la importancia que tiene la protección inmediata de la niña o niño y en el cambio del funcionamiento familiar. Esto ha traído dos perspectivas principales en términos de intervenciones: por un lado la ubicación de la niñez en hogares sustitutos y otra dirigida hacia la atención sobre las personas adultas.

No obstante, consideramos que esta interpretación para acercarse al problema no es suficiente. Ese sujeto que se siente o se le hace sentir culpable evidencia resistencia a dar cuenta en un proceso terapéutico de su vínculo relacional con el hecho y con la niña o niño. En la situación de maltrato infantil, se concluye que los progenitores son incapaces de cumplir con su función parental. Esta percepción trajo que las familias, como plantean algunos autores y autoras, se sientan controladas y juzgadas más que escuchadas (Cirillo y Di Blasio, 1991; Linares y Coletti, 1997). Ávila (2008) expone que la práctica terapéutica que ha estado apoyada en discursos en los que subyace una función de control y represión, no permite la escucha del sujeto, no da lugar para la palabra. Por otro lado, la niña o el niño maltratado muestra una distancia o sobre involucración socio afectiva con la o el terapeuta que puede provocar una relación intrusiva o desorganizada. Lo expuesto hace necesario que se module la distancia y cercanía con la niña o niño para proporcionarle un espacio seguro y de escucha que facilite el apalabrar su experiencia.

La comunidad articula su propia demanda sobre la falta de organización y de concertación en la visión sobre los servicios de los diferentes niveles institucionales y gubernamentales para proponer respuestas a la situación de violencia que vive la niñez (Barudy, 1998). Esta espera que los profesionales de la conducta actúen con firmeza y eficacia para asegurar la vida y seguridad de la niña o niño. Cuando esto no ocurre, se evidencia que la trabajadora social o el terapeuta no han sabido realizar su quehacer de ver, 
escuchar y entender lo que ocurre y que por ende han actuado de manera inapropiada.

\section{Trasfondo de la terapia de juego}

Los aspectos señalados previamente nos permiten la reflexión crítica sobre la atención y desatención de la niñez en situación de desprotección, facilitándonos crear como estrategia terapéutica un espacio de juego en el que participan las personas adultas juntos a la niña o el niño. En este espacio desde un enfoque psicodinámico a partir de la experiencia de jugar, se presta atención al elemento relacional que permite al sujeto y al niño o niña dar cuenta de la experiencia de maltrato. El acercamiento al niño o niña, junto a su familia a través del juego no es novedoso. En la práctica clínica han existido terapeutas que han incorporado tal perspectiva en su quehacer, en el que su meta ha sido apoyar a los miembros de la familia a mirar su queja, síntoma o sufrimiento (Amores, 2000; Browfield, 2003; Gil, 2003; Guerney, 2003; Munns, 2003).

Gil (1994) integró elementos de la terapia de juego y de la terapia de familia para cuestionar las separaciones rígidas entre ambos modelos terapéuticos. Esta observaba que el juego desactiva la resistencia de los adultos que no querían hablar y que a través de este asumían la palabra. Especificaba que el juego ofrecía oportunidades mutuas (adultos y niñez) para reír, divertirse y crear (Gil, 1994). Por otro lado, en 1964 se creó la terapia filial debido a su frustración con los modelos que se utilizaban en las décadas de los años 50 y 60 para acercarse a los problemas emocionales de la niñez (Guerney, 2003). En ésta terapia se postuló la idea de capacitar a padres y madres para que jugaran con sus hijos e hijas de manera terapéutica; y se dio cuenta de que el vínculo entre madre, padre y descendencia, a pesar de los problemas que solían existir en la relación era tan fuerte que sustentaba la relación en cierta forma (Guerney, 2003). La psicóloga Ann Jernberg concibe lo que se conoce como terajuego que es una forma estructurada de terapia de juego que intenta mejorar la vinculación afectiva entre madre, padre e hijos (Munns, 2003). El énfasis principal de este enfoque está ligado a la interacción en el juego; en primer lugar entre el o la terapeuta y el niño o niña, mientras observan sus progenitores, y después directamente entre éstos y su descendencia. 


\section{Perspectiva teórica}

La construcción teórica básica de nuestra posición terapéutica está anclada principalmente en el trabajo de John Bowlby y Francoise Dolto que mantuvieron una mirada en la relación temprana de la niñez junto a su familia. Bowlby (1988) expuso una teoría sobre el desarrollo afectivo de los sujetos. Sostuvo que el apego es un lazo afectivo que una persona forma hacia otra y que perdura a través del tiempo. Tiene a nivel psicológico una función de seguridad física y emocional. Se establece desde las etapas tempranas del desarrollo aunque es más evidente después de los ocho o nueve meses de edad. Para la infancia temprana este proceso es vital, porque necesita cuidados durante un largo período (Bowlby, 1988).

Un patrón de apego seguro permite a la niñez adaptarse a situaciones estresantes modulando sus conductas de apego y procesando la información conflictiva. Los vínculos deben ser de tal calidad que aseguren una vivencia emocional permanente expresada en comportamientos y discursos que consideren a cada miembro de la familia como un otro legítimo, en un proceso de co-existencia permanente (Bowlby, 1988). Por otro lado, Dolto (1996) expone que la niña o el niño necesitan de personas adultas que asuman la función paterna y materna para construirse y añade que un continuo necesario para esa construcción es el afecto. Esta autora sostiene que al infante le hace falta el alimento calórico en igual medida que el alimento afectivo, un clima de intercambio y de seguridad resulta igualmente necesario (Dolto, 1998). Las funciones en el ámbito familiar son sostener y acercar al infante para evitar su angustia y en esa mediación significativa con las instancias paternas y maternas se instale su subjetividad. Dolto (1998) expone que la familia tiene un papel crucial en la protección de la niñez, que va desapareciendo a medida que la niña o el niño van creciendo. Además, enuncia las primeras prohibiciones para que conozca la ley y las reglas que lo introducen en lo social y la conquista de la autonomía. Las personas adultas que demandan del niño o niña la sumisión que impone reglas injustas y de manera coercitiva no permiten el desarrollo o conquista de la autonomía. La prohibición adecuadamente expuesta no implica violencia, ni frustrar al niño o niña.

En 1978 Dolto crea un programa preventivo que se conoce como la Casa Verde, donde se recibía a la niñez menor de cuatro años junto a sus adultos tutelares (Dolto, 1998). El objetivo de la Casa Verde era acoger y emprender 
un acercamiento que facilitara la mirada a la autonomía y la socialización. Los padres y madres observan y comparten experiencias e inquietudes con otros padres, madres, abuelos, abuelas y otros acompañantes sentados en el mismo espacio en que juega la niña o el niño. En la Casa Verde se rechazó todo control y normativa pedagógica (Binet, 2000). Consideraba que a partir del momento en que la reunión se convierte en un lugar en el que todo el mundo habla sin temor de ser juzgado, ni castigado, el grupo hace que todos los participantes hagan intercambios significativos y avancen (Dolto citada en Binet, 2000). La atmósfera en la Casa Verde se caracterizaba por un trato distintivo, tranquilizador y armonioso. La población infantil y sus familias era muy heterogénea, lo que facilitaba el aprendizaje de la tolerancia. Dolto (1985) expuso que la diversidad obliga a unos y otros a colaborar entre todos y promover el respeto a cada uno. Su argumentación a favor de la relación parental filial en un mismo espacio psicoeducativo se convirtió en una referencia clara y directa en este trabajo.

Basado en lo expuesto anteriormente, se construyeron los siguientes postulados considerados fundamentales para el proceso terapéutico:

1. El desarrollo de un apego seguro entre la niña o el niño - la figura de apego parental es un mecanismo protectivo contra los malos tratos en la niñez.

2. Las familias necesitan poder expresar sus sentimientos de odio, celos y enojo de un modo abierto y directo, del mismo modo en que se permiten expresar el afecto y la seguridad.

3. Las reglas rígidas, injustas y coercitivas tienden a hacer a la niñez temerosa, reservada y a frustrar su autonomía.

4. El padre o la madre que utiliza la expresión violenta para enunciar las prohibiciones transita por el sufrimiento igual que el niño o niña.

5. El restablecimiento de la vinculación afectiva entre el niño o niña y el padre, madre o personas adultas tutelares en función parental proporciona una base segura para ambos desde la cual explorarse a si mismos y su ambiente. 


\section{Proceso terapéutico}

Para llevar a cabo la terapia de juego, se ofrece un espacio seguro y libre de prejuicio para exploración de la relación vincular. La atmósfera es de respeto y en la que el juego asume la función de sostén terapéutico. El o la terapeuta reconoce la angustia del padre o madre y su resistencia-confianza a explorar el maltrato hacia su descendencia. También observa los pasos que va dando para asumir la experiencia dolorosa. El papel de la o el terapeuta es de coparticipación, por lo cual, no conduce las actividades o juegos ni ofrece instrucciones para el uso del material. Es en las sesiones iniciales que se posibilita la evaluación precisamente por la ausencia de directrices. Al igual que Dolto, se presta gran atención al ambiente familiar intentando reconocer quién es el que sufre y cómo se estructura la relación entre los miembros de la familia. Las sesiones iniciales se organizan tanto en el espacio de consulta como en la residencia de la familia. La libertad es un punto fundamental en la relación del juego. Los miembros de la familia eligen el material de juego, los roles que asumen y las estrategias para jugar.

En este período promovemos acoger la demanda de escuchar tal y como es articulada por la niña o niño y su familia. En ese sentido brindamos un espacio de juego sin enfatizar en la contribución de las personas adultas al sufrimiento del niño o niña y reconocemos el propio sufrimiento de éstas, ya que podrían evidenciar dificultad para reconocer el maltrato como una realidad que ocurrió. A partir del juego y a través del mismo, los miembros de la familia transitan hacia el período de la aflicción y ambivalencia. Estos comienzan a significar las diferentes situaciones y la relación vincular entre ellos. En sus actividades lúdicas constantemente se categorizan los personajes y las acciones entre los buenos y los malos. El malo es quien se separa, abandona, pega y se muestra distante, por otro lado, el bueno evidencia respuesta afertiva y protege. El padre o la madre juegan en forma directa con la niña o niño pese a sus dudas y temores. El niño o niña intenta evitar la dirección de sus progenitores en el juego mostrando dudas y ambivalencia promoviendo que ellos valoren sus necesidades.

En el período de la apertura, el juego es un recurso para fomentar la cooperación, integración, y la disminución de la agresión. El padre o la madre se comprometen en la relación con el niño o niña. Estos son capaces de reconocer las necesidades el uno del otro y expresar su afecto de manera íntima. Observamos que ocurre la revelación por parte del padre o madre de 
los malos tratos que produjo el sufrimiento del niño o niña. Logran una comprensión significativa de cómo contribuye la revelación a no resistirse a interactuar con el niño o niña de forma afectiva y protectora. De alguna manera, parafraseando a Dolto, el padre o la madre se da a luz a sí mismo, se vuelve responsable de sí mismo. En esta fase la terapeuta interpreta el juego metafórico y simbólico de los miembros de la familia para facilitar la revelación del maltrato.

Luego de este momento crucial en el proceso terapéutico los miembros de la familia comienzan a jugar de manera repetitiva y elaborando sobre los mismos temas. El juego se caracteriza por la animación y la diversión. El contacto físico y personal es recurrente entre los miembros de la familia de tal forma que se agotan. Esta fase la reconocemos como el agotamiento, en la cual los miembros de la familia utilizaron ya todas sus posibilidades y el proceso terapéutico llegó a su fin. La o el terapeuta acoge la angustia y la emoción que la familia experimenta al saber que continuarán jugando sin su presencia.

\section{Presentación del Caso}

Niña de 20 meses producto de una relación casual. Sus progenitores, jóvenes de 18 y 23 años de edad. Removida por el Departamento de la Familia por negligencia grave. El contexto de desprotección se caracterizó por alimentación irregular que implicó deshidratación severa y bajo peso. La niña pasaba mucho tiempo sola y sin supervisión. Muestra un rezago significativo en el lenguaje expresivo no logra dar respuestas a mandatos sencillos de acción. Evidencia dificultad para reclamar atención y pedir algo. Por otro lado, existían intercambios comunicativos inadecuados entre la madre y la niña, que se caracterizaban por las amenazas, críticas y un contacto afectivo mínimo. La custodia provisional fue otorgada a los abuelos paternos. El padre de la niña reside en Estados Unidos y labora para un departamento de seguridad gubernamental. La niña muestra una conducta demandante e irritable y resulta difícil de calmar. Se aferra a su abuela de manera exigente. Evidencia dificultad para organizar y usar el afecto como parte de las relaciones y la interacción con los miembros de la familia.

En las sesiones participaban los abuelos paternos, una tía de 12 años y la niña. La niña se mantuvo alejada del espacio para el juego familiar. No mostraba reciprocidad a las expresiones de afecto de su familia. Paulatinamente se incorporó al juego simulando el cuidado propio con los 
muñecos. Su contacto manipulativo con los muñecos se caracterizaba por pegarle, aferrarse a estos y luego tirarlos a cierta distancia de ella. Sus abuelos la siguen en la simulación, pero de manera juguetona, amigable y cálida. En el juego de la niña predominó una interacción agresiva elaborando la carencia, como expone Dolto del alimento afectivo. En la medida que los abuelos proveyeron un clima de seguridad la aflicción de la niña disminuía reemplazando su juego por uno más animado. El espacio de juego estaba permitiendo el acercamiento de los miembros de la familia.

En este periodo se otorgó custodia provisional a la madre. La niña estaba siendo adiestrada en los hábitos de eliminación. Se evacuó en su ropa poco tiempo después de haberla llevado al baño. Su mamá perdió el control, mientras la iba cambiando le pegaba fuertemente sobre las nalgas y espalda baja produciéndole hematomas. La custodia física nuevamente fue otorgada a sus abuelos paternos. En las sesiones siguientes la niña reflejaba la agresión en su simulación al jugar. En citas sucesivas se mostró retraída y desconfiada. No seguía a su abuela en el juego que elaboraba para ella. El abuelo cedía rápidamente a los deseos de su nieta.

El Tribunal ordenó la participación de la mamá en el proceso terapéutico. Se llevaron a cabo dos sesiones de juego junto a la mamá y la niña. La niña se mantuvo a cierta distancia física de la madre. En la segunda sesión la niña realizaba movimientos repetitivos con su cuerpo que forman parte de una canción recreativa. Entonces acompañamos los movimientos con la estrofa del cántico que dice:

tengo ojos
tengo boca
tengo nariz
tengo mis dos manos
para saludarte a ti

La niña se mantuvo haciendo los movimientos de manera repetitiva. Su mamá le cantó el estribillo diciendo:

tengo ojos

tengo boca

tengo nariz

tengo mis dos manos que

no son para darte a ti 
Intentó acercarse a la niña pero ella rechazó el contacto físico. Los gestos y movimientos que involucraba la canción recreativa cesaron por parte de la niña y comenzó a balancearse de manera intranquila. Exploramos con la mamá sus juegos infantiles. Esta jugaba simulando a la maestra y reproducía con sus muñecas el rol materno. Sugiero a la mamá que simule para su hija alguna forma de juego que ella usaba. Entonces la mamá tomo una muñeca y la llamo por el nombre de la niña y la acaricio, la besó y la movió entre sus brazos. La mamá se involucro de manera muy cálida en la actividad. Después de ese momento, acerco la muñeca a la niña y al poco rato ella comenzó a jugar con la muñeca. Se le interpretó a la mamá que la niña había encontrado consuelo en sus palabras, pues, estaba jugando y moviéndose de manera organizada y tranquila. Las sesiones sucesivas planificadas con la mamá no se llevaron a cabo; pues, ésta no asistió. En las sesiones con los abuelos el juego se transformó nuevamente volviéndose cálido y animado. Una vez se observó que la externalización de la aflicción había ocurrido, finalizó el proceso terapéutico.

A modo de conclusión observamos que el juego asume la función de sostén logrando que una familia apalabre la significación del sufrimiento. Lo que hemos intentado es dar cuenta de la manera en que he realizado la práctica clínica con la niñez temprana expuesta a malos tratos. La trabajadora social Virginia Satir, reconocida terapeuta familiar, independientemente de los acuerdos y desacuerdos que podamos tener desde el punto de vista clínico, dijo en una ocasión que el mejor regalo que podemos recibir de alguien es que nos vea, escuche y entienda; y en esta propuesta terapéutica lo que facilitamos a las familias es verlos, escucharlos y entenderlos. Como señala Ávila (2008), proveer un espacio libre y vivo para el desarrollo de la escucha e intercambios para que la niñez y su familia sean acogidas en su sufrimiento o desamparo, es más valioso que un tratamiento dirigido a eliminar sus síntomas.

\section{Referencias}

Allin, Heather; Mac Millan, Harriet y Wather, Nadine. (2005). Treatment of Child Neglect. A Systematic Review. Canadian Journal of Psychiatric, 50(8), 497-504.

Amores, Sara. (2000). Clínica del niño y su familia: Una perspectiva vincular psicoanalítica. Argentina: Editorial Distal. 
Ávila, Dyhalma. (2008). ¿Hay espacio para la escucha de los niños en instituciones de salud mental? El Amauta, 5, 1-10. Accedido el 31 de marzo de 2008 en http://amauta.upra.edu/dossier5.html

Barudy, Jorge. (1998). El dolor invisible de la infancia. España: Ediciones Paidós Ibérica.

Bear, Judith y Martínez, Colleen. (2006). Child Maltreatment and Insecure Attachment: A Meta Analysis. Journal of Reproductive \& Infant Psychology, 24(3), 187-197.

Binet, Eric. (2000). Francoise Dolto. Unesco Oficina Internacional de Educación. Accedido el 23 de septiembre de 2007 en http://www.ibe.unesco.org/publications/thinkerspdf/doltors.pdf.

Bowlby, John. (1988). A Secure Base: Clinical Applications of Attachment Theory. Londres: Routledge.

Brandell, Jerrold. (2004). Psychodynamic Social Work. New York: Columbia University Press.

Browfield, Richard. (2003). Psychoanalytic Play Therapy. En Charles E. Schaefer (Ed.), Foundations of Play Therapy (1-13). New Jersey: John Wiley.

Cirillo, Stefano y Di Blasio, Paola. (1994). Niños maltratados: Diagnóstico y terapia familiar. España: Ediciones Paidós Ibérica.

Coletti, Maurizio y Linares, Juan Luis. (1997). La intervención sistémica en los servicios sociales ante la familia multiproblemática. España: Ediciones Paidós Ibérica.

Di Lauro, Michelle. (2004). Psychosocial Factors Associated with Types of Child Maltreatment. Child Welfare, 83(2), 69-97.

Dolto, Francoise. (1994). Niño deseado, niño feliz (4ta ed.). Argentina: Editorial Paidós. 
Dolto, Francoise. (1996). La causa de los niños (4ta ed.). Argentina: Editorial Paidós.

Dolto, Francoise. (1998). El niño y la familia: Desarrollo emocional y entorno familiar. Argentina: Editorial Paidós.

Dolto, Francoise. (1999). La educación en el núcleo familiar. Argentina: Editorial Paidós.

Elliot, Katherine y Urquiza, Anthony. (2006). Ethnicity, Culture, and Child Maltreatment. Journal of Social Issues, 62(4), 787-809.

Gil, Eliana. (1994). Play in Family Therapy. New York: Guilford Press.

Gil, Eliana. (2003). Family Play Therapy: The Bear with Short Nails. En Charles E. Schaefer (Ed.), Foundations of Play Therapy (192-218). New Jersey: John Wiley.

Guerney, Louise. (2003). Filial Play Therapy. En Charles E. Schaefer (Ed.), Foundations of Play Therapy (99-142). New Jersey: John Wiley.

Munns, Evangeline. (2003). Theraplay: Attachment - Enhancing Play Therapy. En Charles E. Shaefer (Ed.), Foundations of Play Therapy (156-174). New Jersey: John Wiley.

Nazario, Edna. (2001). Apuntes sobre la clínica. Puerto Rico: Publicaciones Puertorriqueñas.

Ruiz, Isabel y Gallardo, José. (2002). Impacto psicológico de la negligencia familiar (leve versus grave) en un grupo de niños y niñas. Anales de Psicología, 18(2), 261-272.

Sanville, Jean. (1991). The Playground of Psychoanalytic Therapy. New Jersey: Analytic Press.

Suprina, Joffrey y Chang, Catherine. (2005). Child Abuse, Society, and Individual Psychology: What's Power Got to Do with It? Journal of Individual Psychology, 61(3), 250-268. 